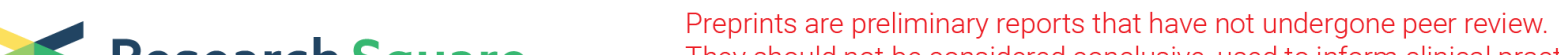 \\ They should not be considered conclusive, used to inform clinical practice, or referenced by the media as validated information.
}

\section{Transcriptome analysis revealed the effect of selenium yeast on improving eggshell quality in aged laying hens}

Zhexi Liu

China Agricultural University

Yutao Cao

China Agricultural University

Yue Ai

China Agricultural University

Xiaonan Yin

Beijing Alltech Biological Products

Lingli Wang

China Agricultural University

Mengyao Wang

China Agricultural University

Bingkun Zhang

China Agricultural University

Keliang Wu

China Agricultural University

Yuming Guo

China Agricultural University

hongbing han ( $\square$ hanhongbing@cau.edu.cn)

China Agricultural University https://orcid.org/0000-0002-6671-719X

\section{Research}

Keywords:

Posted Date: July 19th, 2021

DOl: https://doi.org/10.21203/rs.3.rs-702651/v1

License: (c) (1) This work is licensed under a Creative Commons Attribution 4.0 International License.

Read Full License 


\section{Abstract \\ Background}

Egg internal and eggshell quality were deteriorated in aging laying hens. Improving egg and shell quality can prolong the laying cycle. Although, selenium yeast (SY) has been found with the potential to enhance the laying performance and egg quality, the underlying mechanisms have not been investigated. Therefore, we investigated the effect and molecule mechanism of selenium yeast on egg and shell quality in aged laying hens.

\section{Methods}

Three hundred 76-week-old Jing Hong laying hens were divided into four equal treatments and fed with Se-deficient diet (SD), 0.15, 0.30, $0.45 \mathrm{mg} / \mathrm{kg}$ selenium yeast diet (SY0.15, SY0.30 and SY0.45). At the end of the trial, we evaluated the plasma and tissue selenium content, plasma antioxidant capacity and egg quality. Transcriptomic analysis was performed to investigate the boosting effect of selenium yeast on eggshell quality. The weighted gene co-expression network analysis (WGCNA) analysis was performed to screen out the key candidate genes related with eggshell quality affected by selenium yeast.

\section{Results}

Dietary selenium yeast supplementation enhanced the eggshell strength $(S Y 0.45)(P<0.01)$ and reduced the shell translucence (SY0.15, SY0.30 and SY0.45) to ameliorate the eggshell quality. Moreover, body Se status and plasma antioxidant capacity were significantly enhanced by selenium yeast supplementation $(P<0.05)$, and showed a dose-dependent effect. Transcriptomic and WGCNA analysis identified some key candidate genes involved in eggshell quality including cell migration inducing hyaluronidase 1 (CEMIP), ovalbumin (OVAL), solute carrier family 6 member 17 (SLC6A17), solute carrier family 13 member 5 (SLC13A5), proopiomelanocortin (POMC) and proenkephalin (PENK). Functional enrichment analysis revealed that selenium may alter the biological process including eggshell mineralization, ion transport and laying period to affect eggshell formation.

\section{Conclusion}

Selenium yeast enhanced eggshell strength and reduced the shell translucence by improving antioxidant capacity, selenium status, and regulating the processes of shell mineralization, ion transport and laying period. These findings provide a novel molecule biomarker for affecting eggshell quality regulated by selenium.

\section{Background}


Selenium (Se) is a pivotal micronutrient for animals and humans, and plays vital roles in antioxidant and redox state regulation, immune response, reproductive and multifarious specific metabolic processes [1]. In last few decades, worldwide researchers and nutritionists have studied this essential nutrient with pleiotropic aspects. In general, the function of Se was executed as selenoprotein, which is formed by selenocysteine ( $\mathrm{Sec}$ ) inserted [2]. To date, 25 human selenoprotein genes [3] and 24 confirmed avian selenoprotein genes [4] have been identified.

The current progress in the health impact of upregulation of antioxidant selenoenzymes and cytoprotective properties mediated by Se has been well-known. Dietary Se intakes can affect the synthesis and expression of selenoproteins to modulate deficient or adequate status of Se [5], and appropriate Se status leads to health benefits, such as decreasing mortality and enhanced immune function. Moreover, early research demonstrated that both Se deficiency and excess can cause adverse health effects which accompanied by a U-shaped relationship. Impairments in antioxidant protection, redox regulation, immunity response, and energy production are associated with Se deprivation [6]. Se deficiency may also cause physiological diseases and economic loss in animals and livestock. In chicken, it has been demonstrated that Se deficiency impaired growth performance [7] and led to exudative diathesis [8], nutritional muscular dystrophy[9, 10]. Alarmingly, extra Se supplementation may also have adverse effects on people or animals in adequate Se status, which has been expounded as selenosis. It causes hair and fingernails brittleness, skin and liver damage, and neurotoxicity in livestock [11].

Maintaining egg quality and production performance in aged laying hens for longer laying cycles has aroused an increasing concern in poultry production. In general, the production peak of commercial laying hens usually lasts until 60 weeks, the laying rate and egg quality begin to decline from the middle of production peak period [12]. Aging of hens leads to a deterioration in internal and shell quality of eggs, which is likely to cause the breakage during collection and transport [13]. Thus, overcoming the negative effects of age on egg quality is importance in the late phase of laying cycle. Some previous studies have suggested that the decline in egg quality is associated with nutrients status and oxidant stress in aged laying hens $[13,14]$. Nowadays as a food additive, Se is widely used for enhancing the antioxidants, immunity, health to obtaining Se-enriched byproducts in pigs, chickens, and other livestock. As the organic form of Se, selenium yeast was used frequently because of its characteristics of being wellabsorbed and biological safety $[15,16]$. Substantial studies on boiler and laying hens have shown that selenium yeast supplementation maintained poultry health [17], decreased the egg shape index [18], affected shell thickness [19], enhanced antioxidant status and tissue Se deposition [20]. Meanwhile, previous studies have demonstrated that dietary supplementation selenium yeast can improve the productive performance in aging broiler breeder hens [21]. However, the molecule mechanisms of selenium yeast on egg and shell quality in aged laying hens are poorly understand. In this study, the effects of selenium yeast on egg and shell quality, the optimum dose of selenium yeast in the diet of aged laying hens were investigated. Moreover, the potential molecular mechanism of selenium yeast on eggshell quality was explored by using RNA-seq based global transcriptome analysis. 


\section{Materials And Methods}

\section{Animals, experimental design, diets, and husbandry}

A total 300 76-week-age Jing Hong laying hens were fed with an average basal Se content of 0.056 $\mathrm{mg} / \mathrm{kg}$ corn-soybean diet (the composition and nutrient level of the basal diet were shown in Table S1) for 6 weeks (from 76 to 82 week of age) to investigate the effects of Se deficient on aged laying hens and gain the Se-deficient hens. After Se-consumption Jing Hong laying hens with similar laying rate were randomly allocated to 4 treatment groups with 5 replicates ( 15 chickens per replicate). One group was fed the basal diet only (SD), the remaining groups were supplemented with $0.15,0.30,0.45 \mathrm{mg} / \mathrm{kg}$ selenium yeast (SY) (Alltech, Lexington, KY, USA) for 12 weeks (from 83 to 95 week of age). The Se contents of different diets were shown in Table 1. Batches of the experimental diets were produced every 4 weeks to prevent the feed from mildewing. The hens were housed in an environmentally controlled room maintained at $25^{\circ} \mathrm{C}$ and had a daily lighting schedule of $16 \mathrm{~h}$ light (from 5 am to $9 \mathrm{pm}$ ), $8 \mathrm{~h}$ dark (from 9 pm to $5 \mathrm{am})$.

Table 1

Experiment design

\begin{tabular}{|lllll|}
\hline \multicolumn{4}{l}{ Control } & \multicolumn{2}{l|}{ Selenium yeast } \\
\hline $\begin{array}{l}\text { Group (Supplemented dose, } \\
\text { mg/kg) }\end{array}$ & SD & SY0.15 & SY0.30 & SY0.45 \\
& $(0)$ & $(0.15)$ & $(0.30)$ & $(0.45)$ \\
\hline Measured values & $0.056 \pm$ & $0.211 \pm$ & $0.377 \pm$ & $0.552 \pm$ \\
Mean \pm SEM(mg/kg) & 0.0121 & 0.0145 & 0.0487 & 0.0301 \\
\hline
\end{tabular}

\section{Data and sample collection}

Blood samples ( $8 \mathrm{~mL}$ per laying hen) were taken from the main wing vein and collected into an anticoagulant tube every 2 weeks during the whole feeding period. Plasma was separated by centrifugation at $4^{\circ} \mathrm{C}, 3000 \mathrm{rpm}$ for $10 \mathrm{~min}$ and stored at $-30^{\circ} \mathrm{C}$ for further analysis. After Se supplementation for 12 weeks, 10 randomly chosen laying hens from each dietary treatment were slaughtered after 15-20 hours' post-ovulation. Ovary, magnum, isthmus and shell gland were sampled and frozen in liquid nitrogen immediately. All samples were stored at $-80^{\circ} \mathrm{C}$ prior to analysis. Whilst the other part of the shell gland was fixed in $4 \%$ paraformaldehyde for Hematoxylin-eosin staining.

\section{Measurement of egg quality and translucent egg}

Egg quality was measured both before and after the Se consumption period. In supplementation period, egg quality was measured at 6-week intervals, half of the eggs from each replicate were selected randomly. Eggshell strength, egg weight, egg yolk color, Haugh unit, and albumen height were tested using a digital egg tester (NABEL, DET-6000). Eggshell thickness was measured at the large end, equatorial region, and small end using an Eggshell Thickness Gauge. In the supplementation period, 
another half of eggs from each replicate were selected for the measurement of the translucent egg at 6week intervals. The eggs were stored for $0,7,14$ days at same environment $\left(20^{\circ} \mathrm{C}\right)$ and classified into four score levels, and the scoring method was referenced in this study [22].

\section{Determination of Se content}

To determine the Se content of the feed, plasma, ovary, magnum, isthmus, and shell gland. All samples ( $0.5 \mathrm{~g}-1 \mathrm{~g}$ for feed, ovary, magnum, isthmus, and shell gland and $0.5 \mathrm{ml}-1 \mathrm{ml}$ for plasma) were digested in a mixture of $\mathrm{HNO}_{3}$ and $\mathrm{HClO}_{4}(2: 1)$ for about 2 hours, the mixture was heated at $200^{\circ} \mathrm{C}$ until white fumes appeared. Then $5 \mathrm{~mL}$ hydrochloric acid solution was added in the mixture and heated until white fumes appeared. After the mixture cooled, $20 \mathrm{~mL}$ Ethylene diaminetetraacetic acid (EDTA) was added in the digested sample and adjusted the $\mathrm{pH}$ at 1.5-2.0. After which, $3 \mathrm{~mL}$ 2,3 DiAminoNaphthalene (DAN) was added in the mixture and heated in the boiled water for 5 minutes. After the mixture cooled, $4 \mathrm{~mL}$ cyclohexane was added in the mixture and shook for 8 minutes, then the supernatant was measured by fluorescence method using Hitachi 850 fluorescence spectrophotometer (Tokyo, Japan) [23].

\section{Measurement of antioxidant enzyme activity}

The activity of antioxidant enzymes in plasma can reflect the redox state of the body. The activities of glucokinase and glutathione peroxidase (GSH-Px) and total superoxide dismutase (T-SOD) in plasma samples were determined using the detection kits of GSH-Px and T-SOD (Nanjing JianCheng Bioengineering Institute, Nanjing, China), The activities of total anti-oxidation capacity (T-AOC) in plasma samples were measured by T-AOC Kit (KeyGEN BioTECH, Nanjing, China) following manufacturer's protocol.

\section{Hematoxylin-eosin staining}

For histological assessment, the shell gland was fixed in $4 \%$ paraformaldehyde and embedded in paraffin. Three-micrometer sections were stained with hematoxylin and eosin (H\&E). The entire images were captured by APERIO CS2 (Leica, Germany).

\section{RNA extraction, library preparation, sequencing and RNA- Seq data analyses}

Total RNA was isolated from the shell gland using RNA Isolated Kit (RN4402, Aidlab Biotechnologies, Beijing, China) according to the manufacturer's protocol. Quality and quantity measurements of the extracted RNA were performed using NanoPhotometer (IMPLEN, CA, USA) and a Qubit Fluorometer (Life Technologies, CA, USA), respectively, RNA Integrity Numbers (RIN) were determined using 2100 RNA Nano 6000 Assay Kit (Agilent Technologies, CA, USA). Paired-end transcriptome sequencing was performed using an Illumina Novaseq6000 sequencing platform at Annoroad Biotechnology Co. Inc. (Beijing, China).

\section{Transcriptome Bioinformatic and statistical analysis}

Raw transcriptome reads were quality controlled through filtering out low-quality and adaptor sequences, then we got clean data for further downstream analysis. Clean reads were mapped using Hisat2 to the 
reference genome downloaded from Ensembl (https://asia.ensembl.org/index.html). Quantification of gene expression level was conducted by FPKM (fragments per kilobase of exon per million parts mapping) using the featureCounts package, and differentially expressed genes (DEGs) were accessed using DESeq2 R package. The DEGs were identified with the following parameters: $P$-values $<0.05$ and the fold change value |log2Ratio| $\geq 1$. Gene Ontology (GO) and Kyoto Encyclopedia of Genes and Genomes (KEGG) enrichment analysis were further analyzed in website DAVID (https://david.ncifcrf.gov/). Short Time-series Expression Miner (STEM) was performed (Default parameters) to investigate the dynamic changes in gene expression supplemented by different dose of selenium yeast in shell gland. Weighted gene co-expression network analysis (WGCNA) was performed to identified candidate genes affect eggshell quality regulated by selenium yeast. Pearson correlation $(P<$ 0.05 and $|R|>0.4$ ) was constructed the relationship between modules and Se content. Common genes in significant modules and DEGs were performed to GO enrichment analysis, Sankeycharts exhibited the common pathways between above pathways and DEGs pathways to investigate the process affecting eggshell quality by selenium. The genes in common pathways were performed for Protein-Protein Interaction (PPI) analysis on STRING (https://string-db.org/) website (confidence $=0.7$ ).

\section{Statistical analysis}

Data was presented as mean \pm standard error of the mean (SEM), data was analyzed with one-way ANOVA, followed by Duncan test (SPSS for Windows, version 25; IBM). Statistical differences were considered significant at $P \otimes 0.05$. Phenotypic data presentation was carried out using GraphPad Prism (version 7.0, GraphPad Software Inc, San Diego, CA, USA). Every experiment was repeated at least three times.

\section{Results}

\section{The effect of selenium yeast intakes on plasma Se status and antioxidant enzyme activity}

It is well recognized that plasma and serum selenium can reflect the Se utilization status of body [24], and the decreasing expression of systemic selenoproteins caused by dietary Se deficient led to oxidative stress and oxidative stress-related diseases [25]. In our study, the plasma Se status and antioxidant enzyme activity was measured in aged laying hens during the whole experimental period (Fig. 1). During the consumption period ( $0-6$ weeks), the plasma Se content was significantly declined from 0.237 $\mu \mathrm{g} / \mathrm{mL}$ to $0.068 \mu \mathrm{g} / \mathrm{mL}$ (0-4 weeks) ( $P \otimes 0.01)$ and then remained a stable low status (4-6 weeks) (Fig. 1a). Moreover, redox state in plasma was altered by dietary Se deficient. There were concomitant decreases in plasma T-AOC, GSH-Px and T-SOD activity in the 2-week Se consumption (Pख0.01, $P \otimes 0.01$ and $P \otimes 0.05$, respectively), and then remained relatively stable. Plasma T-AOC activity decreased from $3.716 \mathrm{U} / \mathrm{mL}$ to $1.875 \mathrm{U} / \mathrm{mL}$ (Fig. 1b). The GSH-Px activity was decreased from $3550.145 \mathrm{U} / \mathrm{mL}$ to $1480.000 \mathrm{U} / \mathrm{mL}$ (Fig. 1c). Moreover, it led to a decrease from $270.607 \mathrm{U} / \mathrm{mL}$ to $225.369 \mathrm{U} / \mathrm{mL}$ in plasma T-SOD activity (Fig. 1d). In the supplementation period (6-18 weeks), Se content increased drastically 
( $P \otimes 0.01$ ) with a peak around 6-week supplementation and then reached a plateau in supplementation groups (Fig. 1a). Interestingly, the plasma Se content in SY0.45 group recovered to the level before consumption after 8-week supplementation, while other treatments didn't. Similarly, the selenium yeast supplementation promoted antioxidant capacity in plasma. There was no significant differences in 4week supplementation in the T-AOC activity detection, but it was higher in the SY groups than that in the SD group after 8-week supplementation (P凶0.01) (Fig. 1b). The GSH-Px (Fig. 1C) and T-SOD (Fig. 1d) activity in plasma after 12-week supplementation were significantly higher ( $P \otimes 0.05)$ in the SY0.45 group than the SD group.

\section{Selenium yeast deficiency and supplementation significantly affected egg quality and body Se status}

In the late stage of laying period, changes of nutrients intake have a great impact on laying performance. Some adverse health conditions infertility and reproduction were associated with Se deficiency.

Consistent with our studies, Se deficiency reduced the egg quality. The changes in the egg quality during the consumption period were shown in Table 2. With the increasing week of age and Se consumption, egg weight had an increasing trend $(P=0.06)$, however, eggshell strength was significantly reduced $(P=$ $0.04)$, albumen height, Haugh unit, and yolk color were significantly reduced ( $P \otimes 0.01)$ after 6-week Se consumption. During the supplementation period, eggshell strength was significantly higher in SY0.45 group than that in SD group in 6-week and 12-week supplementation and that in the SY0.15 group in 12week supplementation ( $P \otimes 0.05$ ) (Table S2 and Fig. 2a). Eggs randomly collected after 6 and 12-week supplementation were classified 4 grades according to the degree of shell translucence (Fig. $2 \mathrm{~b}$ and Fig. S1). Supplementation of selenium yeast could reduce the shell translucence no matter how long time eggs stored and the age of laying hens. In addition, selenium yeast supplementation decreased the egg weight, albumen height, and Haugh unit after 12-week supplementation (Table S2).

Table 2

Changes of Egg quality of aged laying hens during the consumption period

\begin{tabular}{|lllllll|}
\hline Period & $\begin{array}{l}\text { Egg } \\
\text { weight } \\
(\mathbf{g})\end{array}$ & $\begin{array}{l}\text { Eggshell strength } \\
\left(\mathbf{k g} / \mathrm{cm}^{3}\right)\end{array}$ & $\begin{array}{l}\text { Albumen } \\
\text { height (mm) }\end{array}$ & $\begin{array}{l}\text { Haugh } \\
\text { unit (U) }\end{array}$ & $\begin{array}{l}\text { Yolk } \\
\text { color }\end{array}$ & $\begin{array}{l}\text { Eggshell } \\
\text { thickness } \\
(\mathbf{m m})\end{array}$ \\
\hline $\begin{array}{l}\text { Before } \\
\text { consumption }\end{array}$ & 61.78 & 3.26 & 6.53 & 79.10 & 6.89 & 0.40 \\
\hline $\begin{array}{l}\text { After } \\
\text { consumption }\end{array}$ & 63.83 & 2.89 & 5.63 & 71.46 & 5.90 & 0.39 \\
\hline SEM & 1.07 & 0.17 & 0.22 & 2.01 & 0.99 & $\varangle 0.01$ \\
\hline$P$-value & 0.06 & 0.04 & $\varangle 0.01$ & $\varangle 0.01$ & $\varangle 0.01$ & 0.40 \\
\hline
\end{tabular}

Furthermore, tissue Se status associated with laying performance including shell gland (Fig. 2c), eggshell (Fig. 2d), magnum (Fig. 2e), isthmus (Fig. 2f) and ovary (Fig. 2g) were detected. The results showed that 
selenium yeast feeding induced a dose-dependent increase in the body Se contents. The dose range which is beneficial for humans and animals is fairly narrow and has been described as a U-shaped curve. In this experiment, a low feeding level was choose to measure whether the shell gland of aged laying hens was in toxicity state by $\mathrm{H} \square \mathrm{E}$ staining. The results showed that there was no degeneration and necrocytosis of the mucosal epithelium cell layer, glands edema or dissolved in the uterus in each group (Fig. $2 \mathrm{~h}$ ). The results indicated that $0.45 \mathrm{mg} / \mathrm{kg}$ selenium yeast supplementation for 12 weeks couldn't cause pathological lesion of aged laying hens.

\section{Dynamic effects of selenium yeast supplementation on gene expression in the shell gland}

A total of 1308 DEGs were assessed in the SY groups compared with the SD group (fold change $\$ 1.00$, $P \otimes 0.05)$ (Table S3). The number of DEGs according to up-regulation or down-regulation were displayed in Fig. 3a. In total, 442 DEGs were shared among three groups, and 296, 144, 130 specific DEGs were harbored in the SY0.15, SY0.30 and SY0.45 group, respectively (Fig. 3b). The biological functions of these DEGs were classified by GO term and KEGG pathway enrichment analysis (Fig. 3c, Fig. 3d and Table S4). DEGs enriched in 44 biological progress, 8 molecular functions, and 18 cellular compartments including cell cycle process (regulation of cell proliferation, regulation of epithelial cell proliferation, and extrinsic apoptotic signaling pathway), female organ development (response to estrogen), and developmental process affected by calcium ions (osteoblast development, positive regulation of bone mineralization and voltage-gated calcium channel activity) which we interested in. Furthermore, based on the KEGG pathway enrichment analysis, ECM-receptor interaction, MAPK signaling pathway, TGF-beta signaling pathway, calcium signaling pathway, glycosaminoglycan biosynthesis, and glutathione metabolism were obtained.

The dose effects of Se on shell gland gene expression were performed by STEM. Total DEGs were clustered into 20 profiles, of which three trend profiles, including 1,2, and 17 showed significant enrichment ( $P \otimes 0.05)$ with the colored block (Fig. 3e), and 17 profiles without color represented the nonsignificant trends (the data was not shown). The expression of 155 genes displayed a reducing trend during the low Se status in profile 2 and the expression of 136 genes displayed an opposite trend in profile 17. In profile 1, the expression of 46 genes showed an initial decrease but a subsequent increase at the Se dose in $0.15 \mathrm{mg} / \mathrm{kg}$.

\section{Transcriptome analysis revealed the potential effects on enhancing eggshell quality supplemented with selenium yeast}

Egg quality traits are important for laying hens, especially in the late laying period. In our study, eggshell strength in the SY0.45 group was significantly higher than that in SD and SY0.15 group. Thus, we used RNA-seq and bioinformatics to explore the potential key genes associated with eggshell strength after selenium yeast supplementation. The Venn diagram showed DEGs from comparisons of SD vs SY 0.45 
and SY0.15 vs SY0.45 (Fig. 4a), 78 DEGs were shared in both two comparisons including CEMIP, SDC3, SLC6A17, OVAL, PER2 and PENK. 73 DEGs were unique in the SY0.15 vs SY0.45 including SLC13A5, $P O M C$ and $C Y F I P 2$, as well as 697 DEGs were unique in the SD vs SY0.45 including EREG, OTOP2, KCNJ2, WNT11, STC2, PTN, SLC26A9, XAF1 and CA2. Functional enrichment analysis results showed that all DEGs were enriched in cell cycle process (regulation of cell proliferation, p53 signaling pathway, cell cycle, and apoptosis), follicle development (regulation of embryonic development, parturition, progesterone-mediated oocyte maturation, and response to estrogen), eggshell mineralization (calcium signaling pathway, glycosaminoglycan biosynthesis, negative regulation of BMP signaling pathway, and cellular zinc ion homeostasis), metabolism (Glutathione metabolism) (Fig. 4b and 4c). It is interesting to note that trend analysis was performed to reveal the expression patterns of candidate DEGs related to eggshell strength at different selenium yeast dose. Total DEGs were clustered into 8 profiles, of which two significant trend profiles ( $P \otimes 0.05)$, including profiles 1 and 6 (colored block) (Fig. 4d). The expression of 158 genes including $C A 2, S L C 13 A 5$, and OTOP2 decreased with higher dose of Se in profile 1. In profile 6, the expression of 123 genes including PENK, WNT7A, and EREG exhibited an obvious increase with the higher dose of Se.

Network analysis is useful to identify genes that are putative hubs of gene co-regulation. Here, we used WGCNA to explore hub genes related to eggshell strength affected by selenium yeast intake. Due to the eggs we collected were not from corresponding laying hens, linear correlation was used to analyze the relationship between shell gland Se content with eggshell Se content and eggshell strength (Fig. 4e), Se content in shell gland had a positive relationship with egg strength $(P=0.0013)$ and eggshell Se content $(P \otimes 0.01)$. The results of WGCNA analysis showed that these genes were segmented into 3 significant modules, and for each of these modules, the correlation of the eigengene with shell gland Se content was computed (Fig. 4f). The expression of gene in the Memagenta and MEgreen module was positively correlated with the shell gland Se content, while it in MEpurple was negatively correlated (Table S5). Common genes both in significant modules and DEGs were selected to explain the effect of selenium yeast on eggshell strength. Functional enrichment analysis showed that common pathways were implicated in the regulation of cell cycle, cell migration, eggshell mineralization, reproductive organ development and shell gland development (Fig. 4g). The PPI result of genes in common GO pathways showed that candidate genes in previous results including PTN, SDC1, WNT11 and PENK (Fig. 4h).

\section{Discussion}

Avian eggshell is made of columnar calcite crystals which protect the eggs from physical damage and microbial contamination, providing calcium sources for developing embryo [26]. Nevertheless, eggshell quality becomes to deteriorate from the middle phase of production with the characteristics of decreasing eggshell strength, increasing ratio of translucent eggs, and increasing numbers of abnormal eggs, which cause substantial economic losses and impaired animal welfare. Numerous studies have identified that Se is beneficial for health, egg and shell quality of laying hens. However, the potential molecular mechanism of selenium yeast on shell quality has not been elucidated. 
Se deficiency and supplementation are closely related to Se status and antioxidants capacity. In general, plasma Se content and antioxidant enzyme were generally considered as useful biomarkers of both Se status and dietary intake [27]. Antioxidant enzyme including T-AOC and T-SOD acted as the important index for redox state in livestock and poultry. GSH-Px is a Se-dependent enzyme which has abilities for protecting tissues from oxidative damage. In our study, Se deficiency for 6 weeks leads to a significant decrease of Se status and antioxidants capacity in aged laying hens. Consistent with other studies, Se deficiency led to a significant decrease of Se content, GSH-Px [28], T-AOC [29], and SOD [30] activities in plasma in pigs and poultry. In addition, we found that $71.31 \%$ of Se content, $49.60 \%$ of T-AOC, $58.31 \%$ of GSH-Px, and $16.71 \%$ of T-SOD were decreased by Se deficient, respectively. Based on the decline degree of these indicators, Se content in plasma decreased quickly, suggesting that it was more sensitive to Se status, and could be a biomarker of Se status. Selenium yeast supplementation also enhanced the Se status and antioxidant capacity. Consistent with other studies, Se content in plasma and tissues increased with a dose-dependent trends after Se supplementation [31, 32]. After 6-week supplementation, plasma Se content in the SY0.45 group was recovered, suggesting that high dose Se supplementation may relieve the harmful effects caused by Se deficiency. Moreover, the effects of Se deposition in different tissues may be different because of complexity of Se absorption and metabolism [33]. We firstly found that the Se deposition hierarchy in tissues associated with laying are isthmus, magnum, ovary, and shell gland, successively. Meanwhile, the activities of GSH-Px, T-AOC, and T-SOD in plasma increased in supplementation period. The data are in agreement with the results previously published that antioxidant enzyme activities dose-depended increased with selenium yeast supplementation [32, 34, 35]. These findings suggested that Se content and plasma antioxidant enzyme activity can reflect the Se status and intakes, and plasma Se content can be used as an indicator of body Se status.

Studies clearly indicated that selenium yeast has crucial roles in poultry nutrition and productive. Eggshell strength is vital in ensuring the integrity and safety of the egg contents, it tends to deteriorate with the increasing of bird age [36]. In our study, Se deficiency for 6 weeks significantly decreased egg quality in aged laying hens. It has been reported that Se deficiency is detrimental to bone microarchitecture possibly through decreasing antioxidant capacity [37]. Thus, these results suggested that oxidative stress caused by Se deficiency [38] may decrease eggshell strength in conjunction with age. Moreover, $0.45 \mathrm{mg} / \mathrm{kg}$ selenium yeast supplementation for 12 weeks significantly elevated egg quality including increasing eggshell strength, and decreasing egg weight and translucent eggs. Consistent with previous studies, eggshell breaking strength was significantly increased after high does selenium yeast supplementation $[39,40]$. Meanwhile, translucent eggshell was a problematic issue to affect eggshell appearance and decrease the commercial value of eggs. The reason of eggshell translucent was still unknown, it was inferred to be associated with variations of eggshell membrane [41]. The decreasing of translucent eggs suggested that selenium yeast supplementation may increase the antioxidant capacity of eggs to ameliorate the decline of egg quality. Moreover, increasing egg weight in the last phase of laying cycle is another of the problematic issues [42-44], large eggs are more difficult to handle and more prone to break during transport and collection. The results in our study showed that 
selenium yeast supplementation decreased the egg weight, suggested that selenium yeast supplementation in last stage of laying period played beneficial roles in egg and shell quality.

In our study, the effect of selenium yeast supplementation on shell gland of aged laying hens were obtained by whole transcriptome analysis. The results of transcriptome analysis revealed several novel genes and biological pathways regulates the ions transport, laying period, eggshell calcification and consequently the eggshell formation. Based on the enrichment pathways, molecular functions, and gene expression trends, thirteen genes were identified as potential candidate genes during eggshell formation including CEMIP, SDC3, OVAL, SPP1, SLC6A17, SLC13A5, OTOP2, CA2, POMC, PTN, PENK, WNT11 and EREG.

The growing crystals interact with the shell organic matrix to form a highly ordered microstructure during shell calcification. Thus, the organic matrix interacts with minerals plays a key role in eggshell formation. We paid particular attention to genes involved in shell mineralization, including CEMIP, SDC3, OVAL, SPP1. CEMIP involved in glycosaminoglycan metabolism and calcium release from endoplasmic reticulum [45]. The up-regulated CEMIP expression ( $\log _{2}$ fold change $>2$ in both two comparison) might suggest that high dose selenium yeast supplementation can promote calcium release for eggshell formation. SDC3 protein possesses domains containing some potential glycosaminoglycan attachment sites [46]. Glycosaminoglycan is widely thought to regulate mineral deposition and determine the properties of eggshell [47]. Thus, we inferred that the up-regulation of SDC3 may play an active role in eggshell formation. OVAL is an abundant eggshell matrix protein binding calcium and plays an active role in carbonate formation [48], in coherence with our results, the up-regulation of OVAL might improve the eggshell formation. SPP1 was known as osteopontin, has mineral-binding domains [49] which involved in calcium metabolism and calcium carbonate precipitation [47] for eggshell calcification. Consistent with other studies, the up-regulation of SPP1 occurred in eggshell calcification period [50, 51]. Similarly, the pathways DEGs enriched in including calcium signaling pathway, glycosaminoglycan biosynthesis and positive regulation of bone mineralization, were also closely associated with eggshell calcification and formation. Hence, the results suggested that selenium yeast supplementation induced shell calcification by regulating the expression of CEMIP, SDC3, OVAL, SPP1 and other candidate genes beneficial for eggshell formation.

The chicken eggshell is a highly ordered structure and is mainly composed of calcium carbonate. Furthermore, the eggshell formation process requires a large amount of calcium $\left(\mathrm{Ca}^{2+}\right)$ and bicarbonate $\left(\mathrm{HCO}_{3}{ }^{-}\right)$, so the ions transport plays a crucial role. Some DEGs and pathways associated with ions transport were screened out in our study, including SLC6A17, SLC13A5, OTOP2, CA2, voltage-gated calcium channel activity and calcium signaling pathway. SLC6A17 and SLC13A5 as solute carrier family, plays an important role in transporting ions across cell membranes for synthesis of eggshell formation, it had been reported that increasing SLC6A17 expression plays an alanine transport role during eggshell formation, while SLC13A5 plays a more important role in the initiation of synthesis of eggshell formation [52]. The carbonate ion required for eggshell synthesis is catalyzed by carbonic anhydrases (CA), and uterine glandular cells possess the carbonic anhydrase activity sites. Growing evidence has 
demonstrated that high level of $C A 2$ expression play a pivotal role for conversion of intracellular $\mathrm{CO}_{2}$ to $\mathrm{HCO}_{3}{ }^{-}$in chicken $[53,54]$. As a member of the otopetrin gene family, OTOP2 gene may have similar functions to $O T O P 1$, which regarded as modulator of cellular calcium influx [55], to transport calcium across the uterine epithelium for eggshell calcification [56]. Our findings inferred that SLC6A17, SLC13A5, OTOP2 and $C A 2$ are the regulators of ions transport affect by selenium yeast.

Moreover, the eggshell formation also depends upon numerous physiological adaptations and processes by the uterine cells, as well as reproductive hormones. In our study, functional enrichment analysis showed that selenium yeast supplementation may affect the response to estrogen, regulation of cell proliferation, regulation of epithelial cell proliferation, and extrinsic apoptotic signaling pathway. POMC plays a role in stimulating the release of cortisol hormone and its up-regulate expression during the mineralization period [52]. Consistent with other studies, a higher expression of POMC was observed in a hard shell egg group [53]. PTNis a developmentally-regulated growth factor and its expression is induced by estrogen [57], PTN and estrogen were reported with a pivotal role in eggshell formation [52]. These findings suggested that selenium yeast may enhance the expression of reproductive hormones to affect the eggshell formation and quality. Indeed, WGCNA analysis also identified some candidate genes which affected the eggshell formation regulated by selenium yeast, including PENK, WNT11, EREG. These upregulation DEGs had been reported in previous studies which had a vital role in eggshell formation [52].

Previous studies have found that trace elements manganese and zinc enhanced eggshell strength by improving biosynthesis of glycosaminoglycan [58] and affecting carbonic anhydrase activity [59], respectively. An increasing number of studies had explored that selenium has a good effect on eggshell quality of laying hens. However, studies on the molecular mechanism of selenium yeast affecting eggshell quality is limited. To date, it is hypothesized that selenium exhibits beneficial regulation on eggshell quality may be directly involved in the process of regulating eggshell formation or the interaction between trace elements. Overall, based on biological functions of the DEGs, we hypothesize that the molecular pathways impacted by selenium on aged laying hens are those related to eggshell mineralization, hormone regulation and ion transduction, suggesting that selenium might play a beneficial role in eggshell formation. Despite that, additional studies are needed to draw conclusive remarks about the molecular mechanisms modulated by selenium yeast in accurate phases of eggshell formation.

\section{Conclusion}

In conclusion, the dietary selenium yeast supplementation improved eggshell strength and reduced eggshell translucence in aged laying hens. Moreover, the plasma Se content, plasma antioxidant enzyme activity, tissue Se content were dose depended on the change of dietary selenium yeast intakes. The plasma Se content could be a biomarker to detect Se status. Transcriptomic analysis revealed that selenium affected eggshell quality through the process of eggshell mineralization, ion transport, and laying period. CEMIP, OVAL, SLC6A17, SLC13A5, POMC and PENK were identified as candidate genes affecting eggshell quality regulated by selenium.

Page $12 / 24$ 


\section{Abbreviations}

Se: Selenium; SY:Selenium yeast; SD:Selenium deficiency; Sec:Selenocysteine; $\mathrm{HNO}_{3}$ :Nitric acid; $\mathrm{HClO}_{4}$ :perchloric acid; GSH-Px:Glutathione peroxidase; T-SOD:Total superoxide dismutase; T-AOC:Total anti-oxidation capacity; H\&E:Hematoxylin and eosin; FPKM:Fragments per kilobase of exon per million parts mapping; DEGs:Differentially expressed genes; GO:Gene Ontology; KEGG:Kyoto Encyclopedia of Genes and Genomes; STEM:Short Time-series Expression Miner; WGCNA:Weighted gene co-expression network analysis; SEM:standard error of the mean; PPI:Protein-Protein Interaction

\section{Declarations}

\section{Acknowledgments}

We thank all the members of the Beijing Key Laboratory of Animal Genetic Improvement and State Key Laboratory of Animal Nutrition who make efforts to these experiments.

\section{Authors' contributions}

ZXL carried out the animal experiments, performed sample analysis and wrote the manuscript. YTC performed bioinformatics data analysis. YA took part in animal feeding and assisted with sample collection. XNY provided the selenium resource. LLW and MYW assisted with sample collection. BKZ and YMG designed experiments and contributed to developing conceptual ideas. KLW contributed to developing conceptual ideas. HBH designed experiments, interpreted the results and revised the original draft of manuscript.

\section{Funding}

This study was supported by the National Key R\&D Program of Intergovernmental Key Projects in China (2018YFE0101700) and the National Key R\&D Program in China (2016YFD0501202).

\section{Availability of data and materials}

The datasets used and/or analyzed during the current study are available from the corresponding author on request.

\section{Ethics approval and consent to participate}

The experimental animal protocols for this study were approved by the Animal Care and Use Committee of China Agricultural University (No. AW05060202-1).

\section{Consent for publication}

Not applicable. 


\section{Competing interests}

The authors declare that they have no competing interests.

\section{Author details}

${ }^{1}$ Beijing Key Laboratory of Animal Genetic Improvement, College of Animal Science and Technology, China Agricultural University, Beijing, China. ${ }^{2}$ State Key Laboratory of Animal Nutrition, College of Animal Science and Technology, China Agricultural University, Beijing, China. ${ }^{3}$ National Engineering Laboratory for Animal Breeding, College of Animal Science and Technology, China Agricultural University, Beijing, China. ${ }^{4}$ Key Laboratory of Animal Genetics, Breeding and Reproduction of the Ministry of Agriculture and Rural Affairs, College of Animal Science and Technology, China Agricultural University, Beijing, China. ${ }^{5}$ Beijing Alltech Biological Products (China) Co., Ltd., Beijing, China.

\section{References}

1. Roman M, Jitaru P, Barbante C. Selenium biochemistry and its role for human health. Metallomics. 2014;6(1):25-54.

2. Berry MJ, Banu L, Chen Y, Mandel SJ, Kieffer JD, Harney JW, et al. Recognition of uga as a selenocysteine codon in type-i deiodinase requires sequences in the 3 ' untranslated region. Nature. 1991;353(6341):273-76.

3. Kryukov GV, Castellano S, Novoselov SV, Lobanov AV, Zehtab O, Guigo R, et al. Characterization of mammalian selenoproteomes. Science. 2003;300(5624):1439-43.

4. Li S, Gao F, Huang J, Wu Y, Wu S, Lei XG. Regulation and function of avian selenogenome. Biochimica et Biophysica Acta (BBA) -. General Subjects. 2018;1862(11):2473-79.

5. Howard MT, Carlson BA, Anderson CB, Hatfield DL. Translational redefinition of uga codons is regulated by selenium availability. J Biol Chem. 2013;288(27):19401-13.

6. Combs GF. Selenium in global food systems. Br J Nutr. 2001;85(5):517-47.

7. Xiang L, Li W, Wang L, Cao C, Li N, Li X, et al. The supranutritional selenium status alters blood glucose and pancreatic redox homeostasis via a modulated selenotranscriptome in chickens (gallus gallus). RSC Adv. 2017;7(39):24438-45.

8. Patterson EL, Milstrey R, Stokstad EL. Effect of selenium in preventing exudative diathesis in chicks. Proceedings of the Society for Experimental Biology and Medicine. 1957;95(4): 617.

9. Dam H, Prange I, Sondergaard E. Muscular degeneration (white striation of muscles) in chicks reared on vitamin e deficient, low fat diets. Acta Pathol Microbiol Scand. 1952;31(2):172-84.

10. Dam H, Søndergaard E. Prophylactic effect of selenium dioxide against degeneration (white striation) of muscles in chicks. Experientia. 1957;13(12):494.

11. Thiry C, Ruttens A, De Temmerman L, Schneider Y, Pussemier L. Current knowledge in species-related bioavailability of selenium in food. Food Chem. 2012;130(4):767-84. 
12. Thiruvenkadan AK, Panneerselvam S, Prabakaran R. Layer breeding strategies: an overview. World's Poultry Science Journal. 2010;66(3):477-502.

13. Molnar A, Maertens L, Ampe B, Buyse J, Kempen I, Zoons J, et al. Changes in egg quality traits during the last phase of production: is there potential for an extended laying cycle? Br Poult Sci. 2016;57(6):842-47.

14. Keshavarz K. The effect of different levels of vitamin $\mathrm{c}$ and cholecalciferol with adequate or marginal levels of dietary calcium on performance and eggshell quality of laying hens. Poultry Sci. 1996;75(10):1227-35.

15. Rayman MP. The use of high-selenium yeast to raise selenium status: how does it measure up? Brit $J$ Nutr. 2004;92(4):557-73.

16. Guo C, Hsia S, Hsiung D, Chen P. Supplementation with selenium yeast on the prooxidantantioxidant activities and anti-tumor effects in breast tumor xenograft-bearing mice. The Journal of Nutritional Biochemistry. 2015;26(12):1568-79.

17. Lu J, Qu L, Shen MM, Wang XG, Guo J, Hu YP, et al. Effects of high-dose selenium-enriched yeast on laying performance, egg quality, clinical blood parameters, organ development, and selenium deposition in laying hens. Poultry Sci. 2019;98(6):2522-30.

18. Lin X, Yang T, Li H, Ji Y, Zhao Y, He J. Interactions between different selenium compounds and essential trace elements involved in the antioxidant system of laying hens. Biol Trace Elem Res. 2020;193(1):252-60.

19. Baylan M, Canogullari S, Ayasan T, Copur G. Effects of dietary selenium source, storage time, and temperature on the quality of quail eggs. Biol Trace Elem Res. 2011;143(2):957-64.

20. Han XJ, Qin P, Li WX, Ma QG, Ji C, Zhang JY, et al. Effect of sodium selenite and selenium yeast on performance, egg quality, antioxidant capacity, and selenium deposition of laying hens. Poultry Sci. 2017;96(11):3973-80.

21. Emamverdi M, Zare-Shahneh A, Zhandi M, Zaghari M, Minai-Tehrani D, Khodaei-Motlagh M. An improvement in productive and reproductive performance of aged broiler breeder hens by dietary supplementation of organic selenium. Theriogenology. 2019;126(279- 85.

22. Wang DH, Chen H, Zhou RY, Huang CX, Gao HX, Fan BL, et al. Study of measurement methods on phenotype of translucent eggs. Poult Sci. 2019;98(12):6677-83.

23. Liao X, Lu L, Li S, Liu S, Zhang L, Wang G, et al. Effects of selenium source and level on growth performance, tissue selenium concentrations, antioxidation, and immune functions of heat-stressed broilers. Biol. Trace Elem. Res. 2012;150(1-3): 158 - 65.

24. Longnecker MP, Stram DO, Taylor PR, Levander OA, Howe M, Veillon C, et al. Use of selenium concentration in whole blood, serum, toenails, or urine as a surrogate measure of selenium intake. Epidemiology. 1996;7(4):384-90.

25. Akahoshi N, Anan Y, Hashimoto Y, Tokoro N, Mizuno R, Hayashi S, et al. Dietary selenium deficiency or selenomethionine excess drastically alters organ selenium contents without altering the 
expression of most selenoproteins in mice. The Journal of Nutritional Biochemistry. 2019;69(120 29.

26. Le Roy N, Combes-Soia L, Brionne A, Labas V, Rodriguez-Navarro AB, Hincke MT, et al. Guinea fowl eggshell quantitative proteomics yield new findings related to its unique structural characteristics and superior mechanical properties. J Proteomics. 2019;209:103511.

27. Thomson DC. Assessment of requirements for selenium and adequacy of selenium status: a review. Eur J Clin Nutr. 2004;58(3):391-402.

28. Liu Y, Zhao H, Zhang Q, Tang J, Li K, Xia XJ, et al. Prolonged dietary selenium deficiency or excess does not globally affect selenoprotein gene expression and/or protein production in various tissues of pigs. J Nutr. 2012;142(8):1410.

29. Zhang ZW, Wang QH, Zhang JL, Li S, Wang XL, Xu SW. Effects of oxidative stress on immunosuppression induced by selenium deficiency in chickens. Biol Trace Elem Res. 2012;149(3):352-61.

30. Cao C, Zhao X, Fan R, Zhao J, Luan Y, Zhang Z, et al. Dietary selenium increases the antioxidant levels and atpase activity in the arteries and veins of poultry. Biol Trace Elem Res. 2016;172(1):22227.

31. Li KX, Wang JS, Yuan D, Zhao RX, Wang YX, Zhan XA. Effects of different selenium sources and levels on antioxidant status in broiler breeders. Asian Austral J Anim. 2018;31(12):1939-45.

32. Chen G, Wu J, Li C. The effect of different selenium levels on production performance and biochemical parameters of broilers. Italian Journal of Animal ence. 2013;12(4):211-21.

33. Pan C, Huang K, Zhao Y, Qin S, Chen F, Hu Q. Effect of selenium source and level in hen's diet on tissue selenium deposition and egg selenium concentrations. Journal of Agricultural Food Chemistry. 2007;55(3):1027-32.

34. Pan C, Zhao Y, Liao SF, Chen F, Qin S, Wu X, et al. Effect of selenium-enriched probiotics on laying performance, egg quality, egg selenium content, and egg glutathione peroxidase activity. J Agric Food Chem. 2011;59(21):11424-31.

35. Meng T, Liu Y, Xie C, Zhang B, Huang Y, Zhang Y, et al. Effects of different selenium sources on laying performance, egg selenium concentration, and antioxidant capacity in laying hens. Biol Trace Elem Res. 2019;189(2):548-55.

36. Bain MM, Nys Y, Dunn IC. Increasing persistency in lay and stabilising egg quality in longer laying cycles. What are the challenges? Brit. Poultry Sci. 2016;57(3):330-38.

37. Cao JJ, Gregoire BR, Zeng H. Selenium deficiency decreases antioxidative capacity and is detrimental to bone microarchitecture in mice. J Nutr. 2012;142(8):1526.

38. Messaoudi I, Hammouda F, Heni JE, Baati T, Sa DK, Kerkeni A. Reversal of cadmium-induced oxidative stress in rat erythrocytes by selenium, zinc or their combination. Experimental Toxicologic Pathology. 2010;62(3):281-88.

39. Invernizzi G, Agazzi A, Ferroni M, Rebucci R, Fanelli A, Baldi A, et al. Effects of inclusion of seleniumenriched yeast in the diet of laying hens on performance, eggshell quality, and selenium tissue 
deposition. Ital J Anim Sci. 2013;12(1):e1.

40. Yang Y, Sun Y, Sun B, Zhu Q, Zhang J, Gao P. Effects of selenium yeast supplementation on performance, egg quality, antioxidant and lipid metabolism and their related gene expression of laying hens at late laying period. Chinese Journal of Animal Nutrition. 2018;30(11):4397-407.

41. Wang DH, Li YJ, Liu L, Liu JS, Bao M, Yang N, et al. Management and production: traits of eggshells and shell membranes of translucent eggs. Poultry Sci. 2017;96(2):351-58.

42. Tumová E, Gous RM. Interaction of hen production type, age, and temperature on laying pattern and egg quality. Poultry Sci. 2012;91(5):1269-75.

43. Saunders-Blades JL, Macisaac JL, Korver DR, Anderson DM. The effect of calcium source and particle size on the production performance and bone quality of laying hens. Poultry Sci. 2009;88(2):338-53.

44. Bennett CD. Influence of egg weight on egg breakage in the field. J Appl Poultry Res. 1992;1(4):399402.

45. Evensen NA, Kuscu C, Hoang-Lan N, Zarrabi K, Dufour A, Kadam P, et al. Unraveling the role of kiaa1199, a novel endoplasmic reticulum protein, in cancer cell migration. JNCl-J Natl Cancer I. 2013;105(18):1402-16.

46. Gould SE, Upholt WB, Kosher RA. Syndecan 3: a member of the syndecan family of membraneintercalated proteoglycans that is expressed in high amounts at the onset of chicken limb cartilage differentiation. Proceedings of the National Academy of ences. 1992;89(8): 3271-75.

47. Chien YC, Hincke MT, Vali H, Mckee MD. Ultrastructural matrix-mineral relationships in avian eggshell, and effects of osteopontin on calcite growth in vitro. J Struct Biol. 2008;163(1):84-99.

48. Stapane L, Le Roy N, Ezagal J, Rodriguez-Navarro AB, Labas V, Combes-Soia L, et al. Avian eggshell formation reveals a new paradigm for vertebrate mineralization via vesicular amorphous calcium carbonate. J. Biol. Chem. 2020: A120-14542.

49. Kazanecki CC, Uzwiak DJ, Denhardt DT. Control of osteopontin signaling and function by posttranslational phosphorylation and protein folding. J Cell Biochem. 2010;102(4):912-24.

50. Pines M, Knopov V, Bar A. Involvement of osteopontin in egg shell formation in the laying chicken. Matrix Biology Journal of the International Society for Matrix Biology. 1995;14(9):765.

51. Poyatos Pertiñez S, Wilson PW, Icken W, Cavero D, Bain MM, Jones AC, et al. Transcriptome analysis of the uterus of hens laying eggs differing in cuticle deposition. BMC Genomics. 2020;21(1).

52. Khan S, Wu SB, Roberts J. Rna-sequencing analysis of shell gland shows differences in gene expression profile at two time-points of eggshell formation in laying chickens. BMC Genomics. 2019;20(1).

53. Brionne A, Nys Y, Hennequet-Antier C, Gautron J. Hen uterine gene expression profiling during eggshell formation reveals putative proteins involved in the supply of minerals or in the shell mineralization process. BMC Genom. 2014;15:220. 
54. Wistedt $A$, Ridderstråle $Y$, Wall $H$, Holm L. Age-related changes in the shell gland and duodenum in relation to shell quality and bone strength in commercial laying hen hybrids. Acta Vet. Scand. 2019;61(1).

55. Kim E, Hyrc KL, Speck J, Lundberg YW, Salles FT, Kachar B, et al. Regulation of cellular calcium in vestibular supporting cells by otopetrin 1. J Neurophysiol. 2010;104(6):3439-50.

56. Sah N, Kuehu DL, Khadka VS, Deng Y, Peplowska K, Jha R, et al. Rna sequencing-based analysis of the laying hen uterus revealed the novel genes and biological pathways involved in the eggshell biomineralization. Sci Rep-UK. 2018;8(1):16812-53.

57. Lee JY, Jeong W, Lim W, Kim J, Bazer FW, Han JY, et al. Chicken pleiotrophin: regulation of tissue specific expression by estrogen in the oviduct and distinct expression pattern in the ovarian carcinomas. PLoS One. 2012;7(4):e34215.

58. Zhang YN, Wang J, Zhang HJ, Wu SG, Qi GH. Effect of dietary supplementation of organic or inorganic manganese on eggshell quality, ultrastructure, and components in laying hens. Poultry Sci. 2017;96(7):2184-93.

59. Innocenti A, Zimmerman S, Ferry JG, Scozzafava A, Supuran CT. Carbonic anhydrase inhibitors. Inhibition of the zinc and cobalt $y$-class enzyme from the archaeon methanosarcina thermophila with anions. Bioorg Med Chem Lett. 2004;14(12):3327-31.

\section{Figures}


A

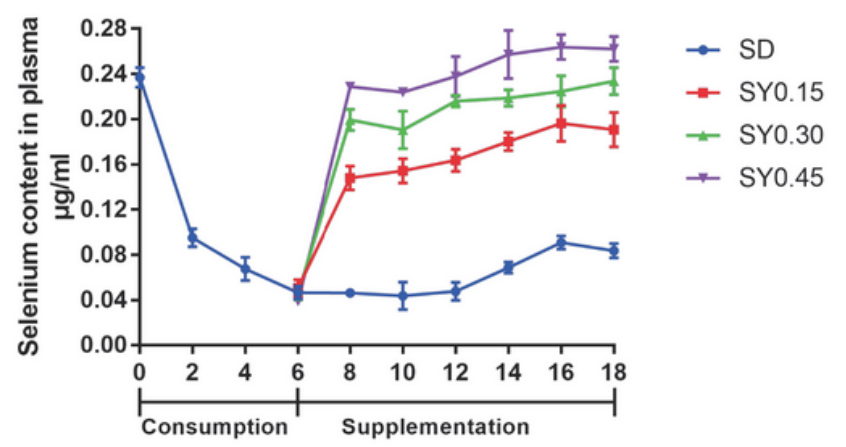

C

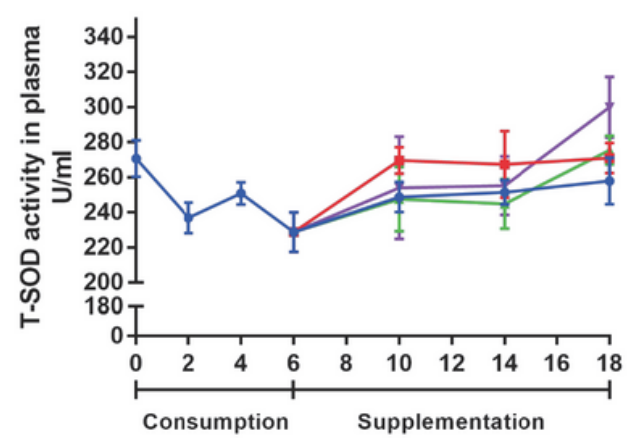

B

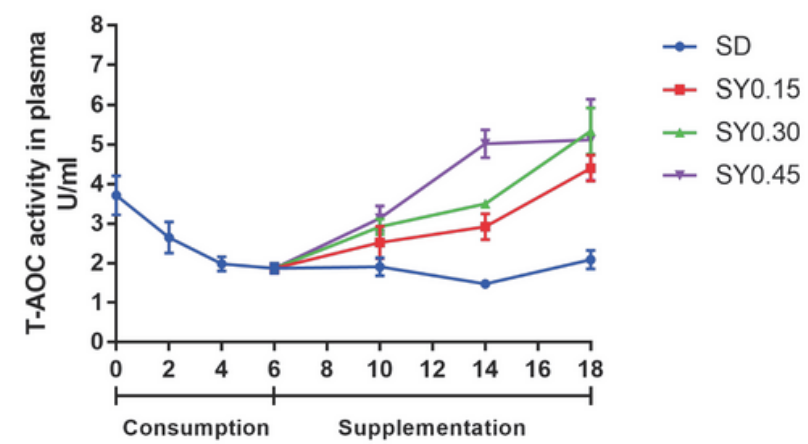

D

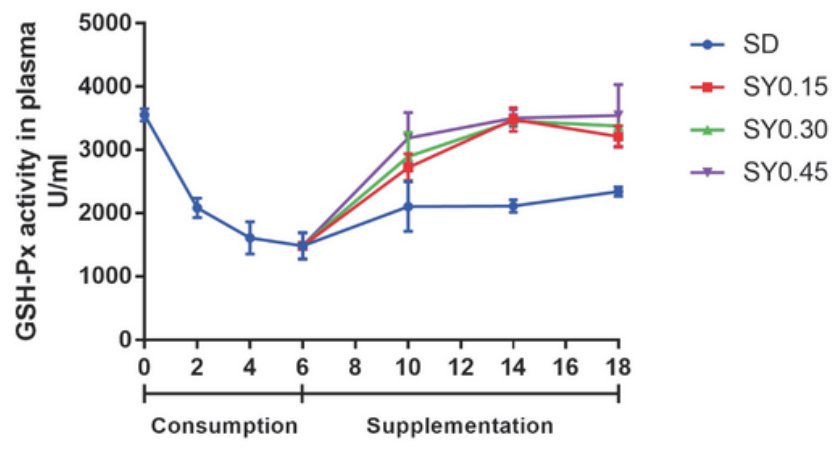

Figure 1

Se status and antioxidant capacity in plasma affected by selenium yeast deficiency and supplementation in aged laying hens during the whole experiment period. (a) Selenium content in plasma in the whole experiment period. (b) T-AOC activity in plasma in the whole experiment period. (c) T-SOD activity in plasma in the whole experiment period. (d) GSH-Px activity in plasma in the whole experiment period. Data are expressed as mean \pm SEM. 
A

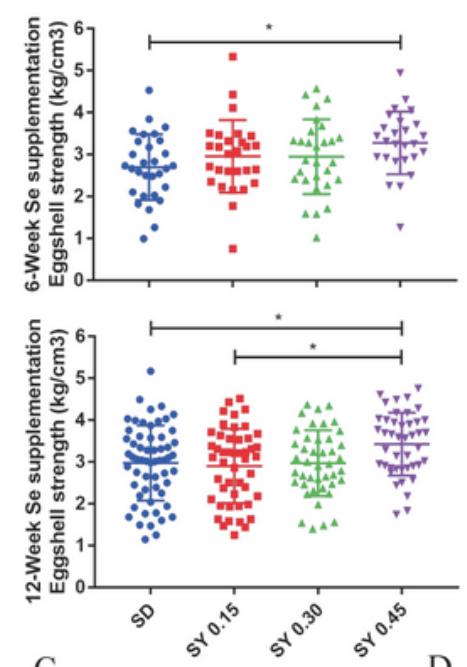

$\mathrm{C}$

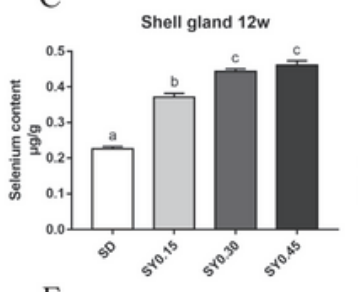

$\mathrm{F}$

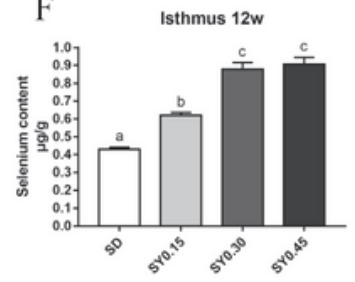

$\mathrm{D}$

G
B
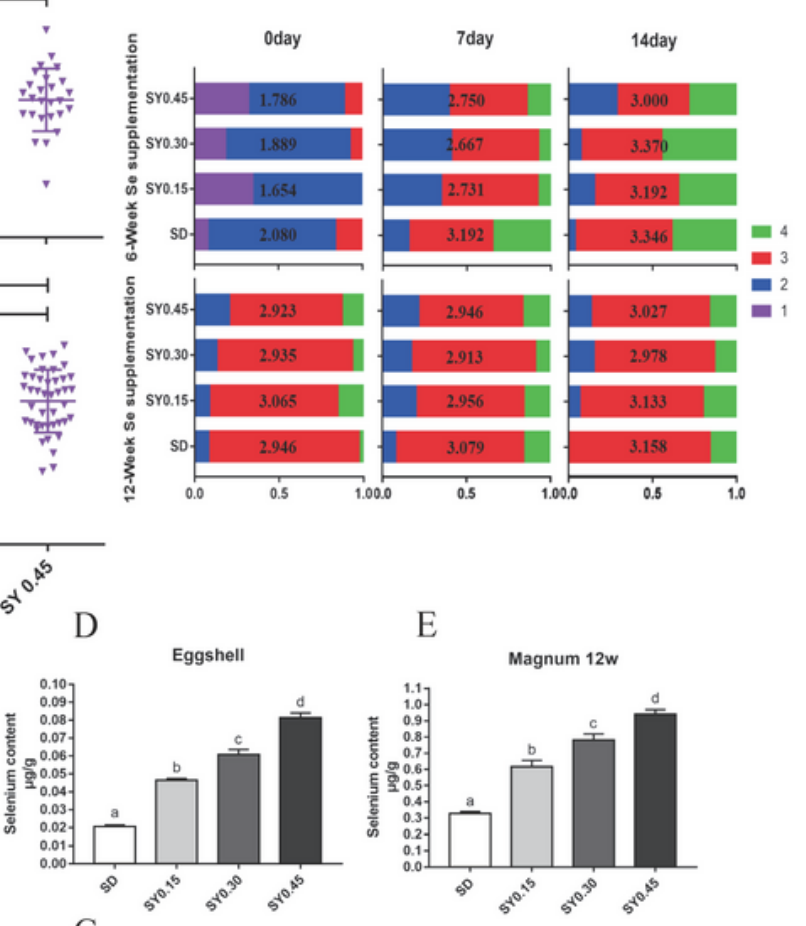

E

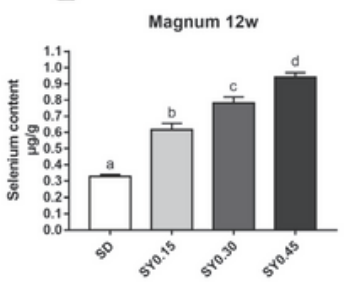

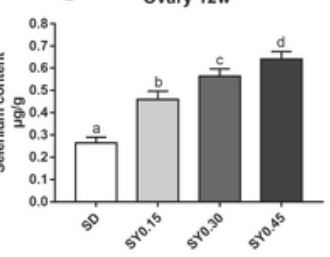

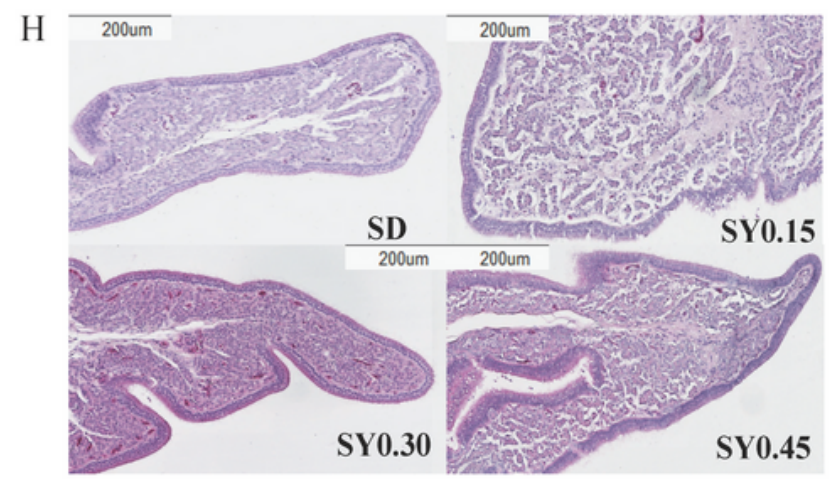

\section{Figure 2}

Eggshell quality and tissue Se status in aged laying hens during the whole experiment period. (a) Eggshell strength during selenium yeast supplementation period. Data are expressed as mean $\pm S E M$. * means $\mathrm{P} \otimes 0.05$. (b) The changes of translucent eggs during selenium yeast supplementation, the average score was shown in the middle of the bar. The data in the horizontal row were detected on different days when egg stored, similarly, the data in the vertical row were detected in different weeks of age. (c) 
Selenium content in the shell gland after selenium yeast supplementation for 12 weeks. (d) Selenium content in eggshell after selenium yeast supplementation for 12 weeks. (e) Selenium content in magnum after selenium yeast supplementation for 12 weeks. (f) Selenium content in isthmus after selenium yeast supplementation for 12 weeks. (g) Selenium content in ovary after selenium yeast supplementation for 12 weeks. Data are expressed as mean \pm SEM. Within each panel, means without a common letter differ at $P \llbracket 0.05$. (h) HखE staining of representative in shell gland after selenium yeast supplementation for 12 weeks $(20 x)$. Scale bar $=200 \mu \mathrm{m}$.

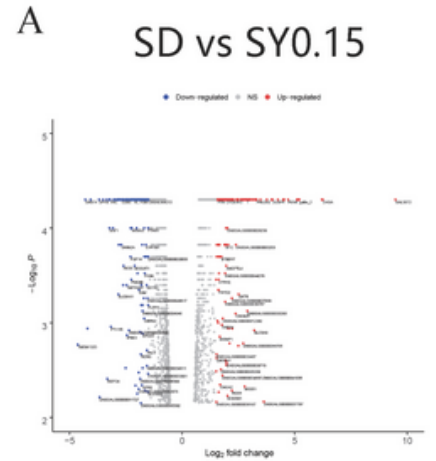

B
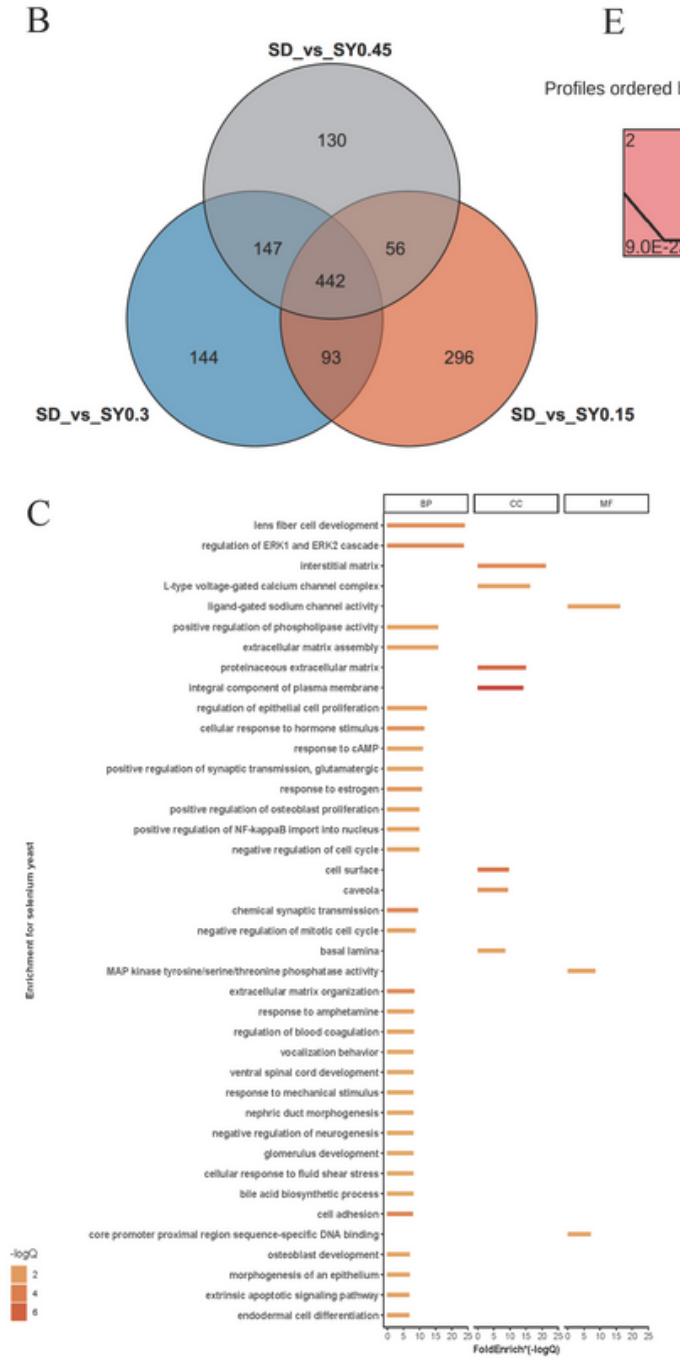

SD vs SY0.30

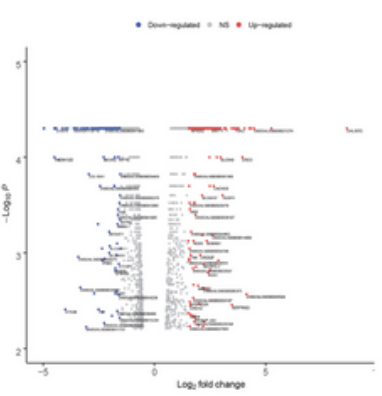

E
SD vs SY0.45

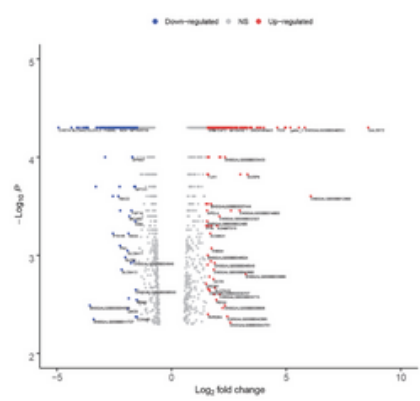

$\therefore$ Lositestromos 


\section{Figure 3}

Transcriptome analysis revealed the effect on gene expression of shell gland in aged laying hens. (a) Scatter plots of DEGs (SD vs. SY0.15, SD vs. SY0.30 and SD vs. SY0.45). Red points represent upregulated genes with a log2 (fold change) $>1$ and $P<0.05$. Blue points represent down-regulated genes with a log2 (fold change) $<-1$ and $P<0.05$. Gray points represent genes showing no significant difference. Fold change $=$ normalized gene expression in the SY0.15, SY0.30 and SY0.45 group / normalized gene expression in the SD group. (b) Venn diagram showing the distribution of DEGs in three comparisons of Se supplementation. The number of DEGs is indicated in the diagram. (c) Gene Oncology enrichment analysis of DEGs identified among the three conditions (SD vs SY0.15, SD vs SY0.30 and SD vs SY0.45). (d) KEGG pathway enrichment analysis of DEGs identified among the three conditions (SD vs SY0.15, SD vs SY0.30 and SD vs SY0.45). (e) Identification of 3 significant gene cluster profiles by STEM. Colored block trend: significant enrichment trend (Pख0.05). The number of genes in each significant cluster was shown after the cluster number. 


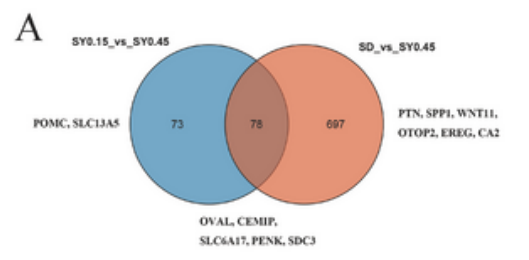

B

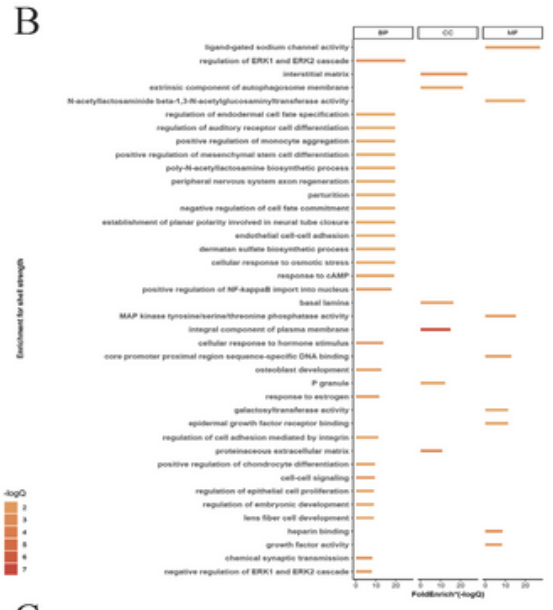

$\mathrm{C}$

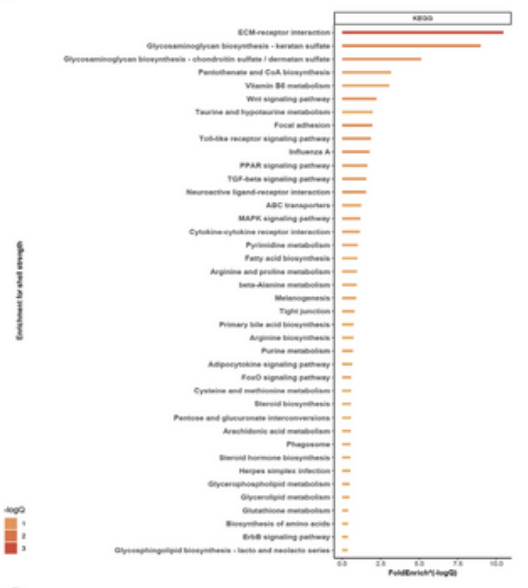

G

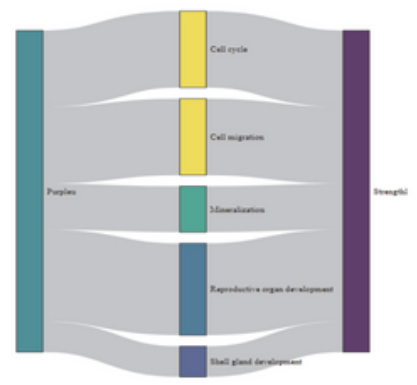

D

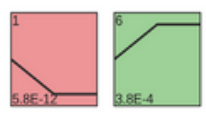

E
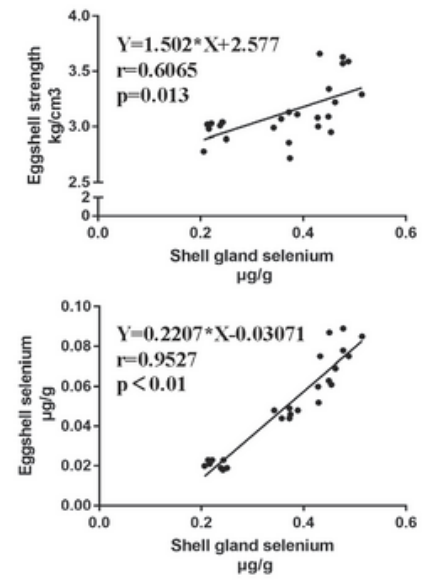

$\mathrm{F}$

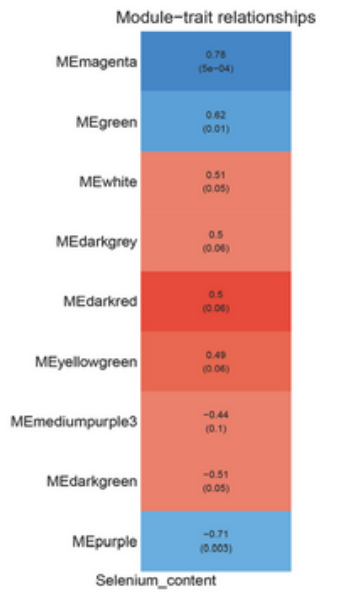

H

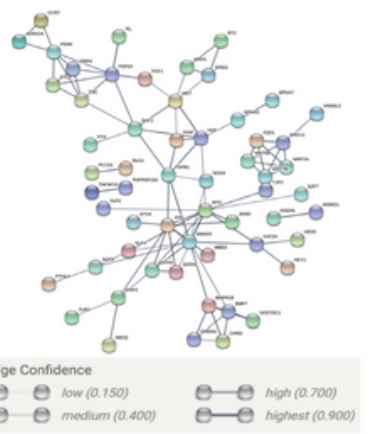

\section{Figure 4}

The potential molecular mechanism of selenium yeast affecting the eggshell strength in aged laying hens. (a) Venn diagram showing the distribution of DEGs associated with eggshell strength. (b) Gene Oncology enrichment analysis of DEGs associated with eggshell strength (SY0.15 vs SY0.45 and SD vs SY0.45). (c) KEGG pathway enrichment analysis of DEGs associated with eggshell strength (SY0.15 vs SY0.45 and SD vs SY0.45). (d) Trend analysis of DEGs associated with eggshell strength expression. 
Colored block trend: significant enrichment trend ( $\mathrm{p} \otimes 0.05)$. (e) The linear correlation between shell gland selenium content with eggshell strength and eggshell selenium content. (f) WGCNA analysis identified specific transcriptional modules related to eggshell strength in aged laying hens after selenium yeast supplementation. (g) Sankeycharts were performed among differential expressed genes in modules and pathways. (h) The PPI network of common genes in modules and DEGs.

\section{Supplementary Files}

This is a list of supplementary files associated with this preprint. Click to download.

- AppendixFig1.pdf

- TableS1.docx

- TableS2.docx

- TableS3.xIsx

- TableS4.xIsx

- TableS5.xIsx 